\title{
Synthesis of Lead Chalcogenide Nanoparticles for Ionizing Radiation Detector
}

\author{
Geehyun Kim,* and Mark D. Hammig* \\ * Department of Nuclear Engineering and Radiological Sciences, University of Michigan, Ann Arbor, \\ MI 48109
}

In the current global situation, the demand for precise and efficient nuclear radiation detection has increased, especially in homeland security and medical imaging applications. The utilization of Nanocrystalline (NC) semiconductor materials, called "quantum dots" which exhibit exploitable properties - such as tunable energy band gap, and charge carrier multiplication - arisen by the strong quantum confinement effect can, in principle, revolutionize the capabilities of radiation detectors by yielding a high-performance detector via a low-cost solution-based fabrication modality. If the NC particles are made uniform across a macroscopic assembly, then excitons of a given multiplicity result in a non-Poissonian carrier population, their number limited only by energy conservation constraints, thus one can potentially quench the statistical counting noise associated with charge carrier creation in the bulk material [1], [2]. Lead chalcogenide ( $\mathrm{PbS}, \mathrm{PbSe}$, and $\mathrm{PbTe}$ ) materials have large Bohr radii of exciton (20 nm, $46 \mathrm{~nm}$ and $50 \mathrm{~nm}$ for $\mathrm{PbS}, \mathrm{PbSe}$ and $\mathrm{PbTe}$, respectively), which enables strong quantum confinement in relatively large NC structures. In addition, high atomic numbers $\left(\mathrm{Z}_{\mathrm{eff}}=56,67\right.$ for $\mathrm{PbSe}$, PbTe respectively) of the lead chalcogenide material would promote the detection efficiency of the detection of incident quanta [3].

Lead chalcogenide ( $\mathrm{PbSe}$ and $\mathrm{PbTe}$ ) $\mathrm{NC}$ particles of different sizes and shapes were synthesized through high-temperature solution-phase route [4], by changing the reaction conditions, such as the reaction temperature, the growing time of the particle and the concentration of precursor reagents and surfactants. Synthesized NC particles were characterized with Transmission Electron Microscope (TEM) and the size and the shape of the NC particles made in various reaction conditions are reported in Table 1. Size and shape of the NC particles in each sample exhibited fairly uniform feature over the sample. The size of the NC particles increased with the reaction temperature and the shape of the NC particle was altered by varying the concentration of precursor reagents and surfactants which arises different controlling power on the particle growth. Notably, higher reaction temperature resulted in the formation of the larger NC particles as shown in Fig. 1-3. Fig. 1(a)-(d) show different size and shape of the PbSe particles synthesized in various reaction conditions. The relative concentration of precursor reagents $(\mathrm{Pb}: \mathrm{Se})$ and surfactants $(\mathrm{Pb}:$ Oleic Acid) affected the shape of the NC particle, of which the growth is differently controlled by the relative concentration of the surfactants and the reaction speed arisen from the different ratio of the precursors. Fig. 2(a)-(c) show high resolution image of star-shaped PbSe NC particles shown from [001] and [111] directions respectively. Spacing between lattice fringes verified the rock salt structure $(\mathrm{Fm} \overline{3} \mathrm{~m})$ of the $\mathrm{PbSe}$ with the lattice parameter $\mathrm{a}=6.13 \AA$. Each arm of the PbSe NC star stretches out to the $<100>$ directions in each axis, as Fig. 2(a) and (b) show the $\{200\}$ lattice fringes on the four arms in the $x-y$ plane. This corresponds with the observation of the $\{220\}$ lattice fringe majority in Fig. 2(c). Fig. 3(a)-(c) show the HRTEM image of PbTe NC particles synthesized in different reaction temperatures. Similarly, the size of the NC particles increased with the reaction temperature. Lattice spacings in each figure match with the distances between (200), or (110) planes in the rock salt structure of the PbTe crystal respectively, with the lattice parameter $\mathrm{a}=6.47 \AA$. Close-packed NC assemblies of lead chalcogenides formed by drop-casting or spin-casting the NC dispersion on various metal contacts, making use of its self-agglomerating nature will be tested for their response to the nuclear radiation. 


\section{References}

[1] V. I. Klimov, J. Phys. Chem. B, 110 (2006) 16827.

[2] G. Kim et al., IEEE Trans. Nucl. Sci. 56 (2009) 841.

[3] G. Kim and M. D. Hammig, IEEE Nucl. Sci. Symp. Conf. Rec. (2009) 1317.

[4] C. B. Murray et al., IBM J. Res. \& Dev. 45 (2001) 47.

[5] This research was supported by U.S. Defense Threat Reduction Agency (DTRA) Basic Research Program, and performed at the EMAL in the University of Michigan. The great help from the Professor Lumin Wang and Dr. Weixing Li is gratefully acknowledged.

TABLE 1. Various synthesis conditions and the corresponding size and shape of the NC particle.

\begin{tabular}{|c|c|c|c|c|c|c|c|}
\hline Precursor ratio & Surfactant ratio & Injection & Growth & Time & Size & Shape & Fig. \\
\hline $\mathrm{Pb}: \mathrm{Se}=1: 1$ & $\mathrm{~Pb}: \mathrm{OA}=1: 2.77$ & $150^{\circ} \mathrm{C}$ & $123^{\circ} \mathrm{C}$ & $3 \mathrm{~min}$. & $7-9 \mathrm{~nm}$ & Spherical & $1(\mathrm{a})$ \\
\hline $\mathrm{Pb}: \mathrm{Se}=1: 3$ & $\mathrm{~Pb}: \mathrm{OA}=1: 4$ & $150^{\circ} \mathrm{C}$ & $123^{\circ} \mathrm{C}$ & $3 \mathrm{~min}$. & $12-14 \mathrm{~nm}$ & Star-shaped & $1(\mathrm{~b})$ \\
\hline $\mathrm{Pb}: \mathrm{Se}=1: 3$ & $\mathrm{~Pb}: \mathrm{OA}=1: 4$ & $180^{\circ} \mathrm{C}$ & $142{ }^{\circ} \mathrm{C}$ & $3 \mathrm{~min}$. & $\sim 20 \mathrm{~nm}$ & Star-shaped & $1(\mathrm{c})$ \\
\hline $\mathrm{Pb}: \mathrm{Se}=1: 3$ & $\mathrm{~Pb}: \mathrm{OA}=1: 4$ & $210^{\circ} \mathrm{C}$ & $165^{\circ} \mathrm{C}$ & $3 \mathrm{~min}$. & $\sim 30 \mathrm{~nm}$ & Star-shaped & $1(\mathrm{~d})$ \\
\hline $\mathrm{Pb}: \mathrm{Te}=1: 1$ & $\mathrm{~Pb}: \mathrm{OA}=1: 4$ & $120^{\circ} \mathrm{C}$ & $120^{\circ} \mathrm{C}$ & $6 \mathrm{~min}$. & $5-7 \mathrm{~nm}$ & Spherical & $3(\mathrm{a})$ \\
\hline $\mathrm{Pb}: \mathrm{Te}=1: 1$ & $\mathrm{~Pb}: \mathrm{OA}=1: 4$ & $135^{\circ} \mathrm{C}$ & $135^{\circ} \mathrm{C}$ & $6 \mathrm{~min}$. & $8-12 \mathrm{~nm}$ & Elliptical & $3(b)$ \\
\hline $\mathrm{Pb}: \mathrm{Te}=1: 1$ & $\mathrm{~Pb}: \mathrm{OA}=1: 4$ & $150^{\circ} \mathrm{C}$ & $145^{\circ} \mathrm{C}$ & $6 \mathrm{~min}$. & $\sim 20 \mathrm{~nm}$ & Spherical & $3(\mathrm{c})$ \\
\hline
\end{tabular}
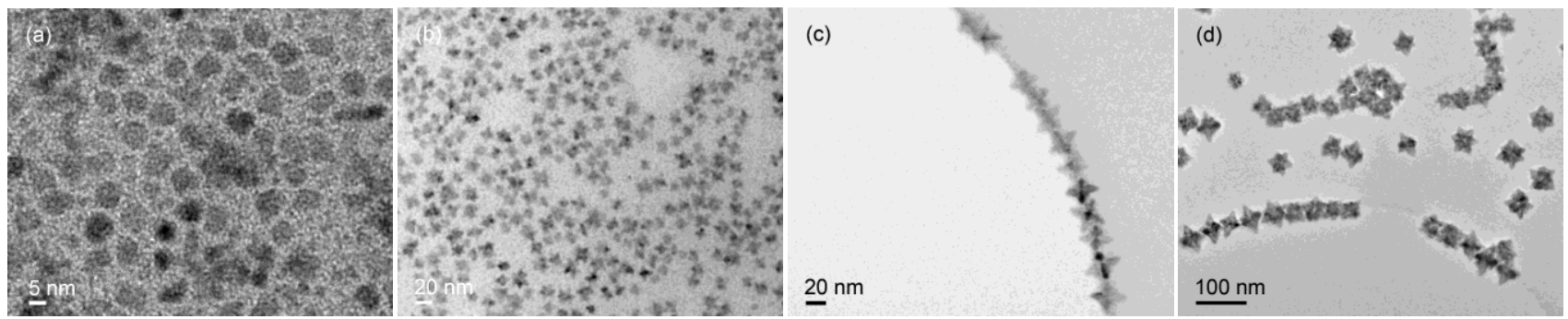

FIG. 1. PbSe NC particles synthesized in various reaction conditions as listed in Table 1.
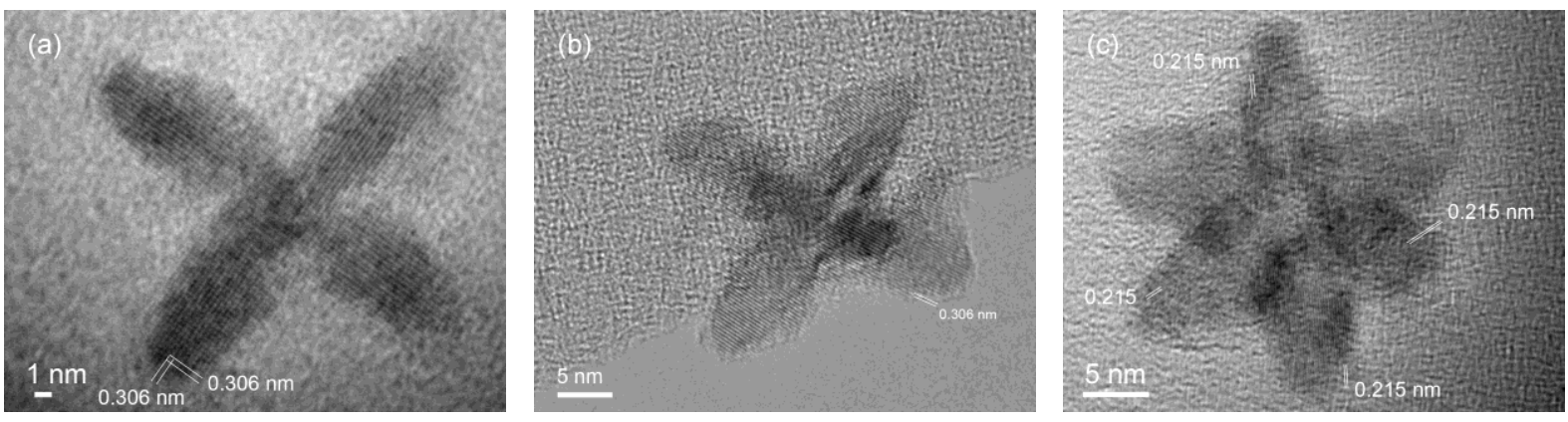

FIG. 2. High resolution images of the star-shaped PbSe NC particle (2a - Fig. 1(b) and 2b, 2c - Fig. 1(c)) observed from [001] direction (2a and 2b) and from [111] direction (2c).
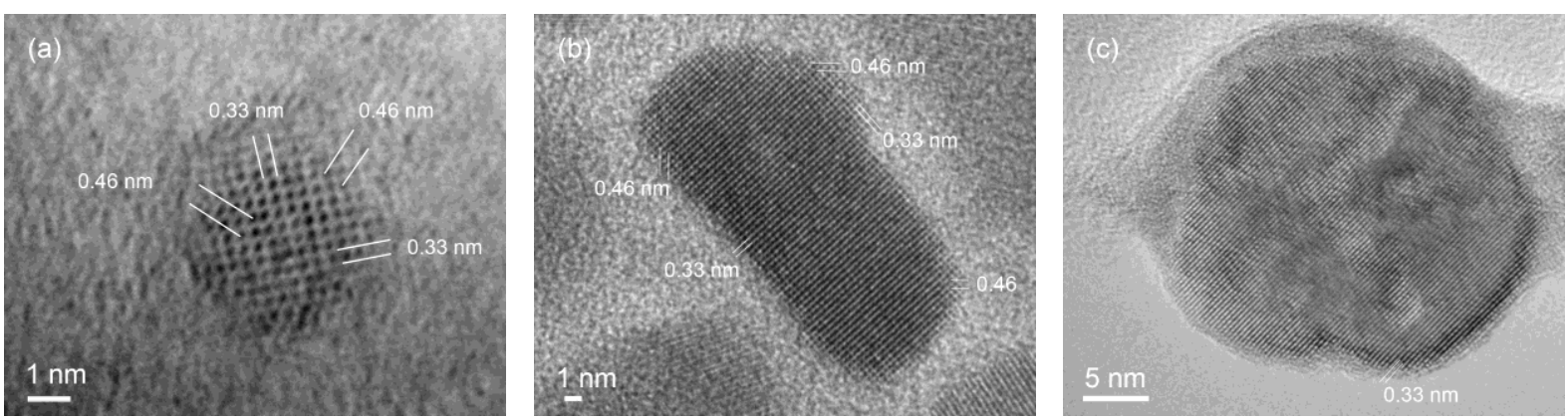

FIG. 3. HRTEM images of single PbTe NC particle synthesized in the reaction conditions as listed in Table 1. 